\title{
COMPACT REPRESENTATIONS BY FINITE-STATE TRANSDUCERS
}

\author{
Mehryar Mohri \\ Institut Gaspard Monge-LADL \\ Université Marne-la-Vallée \\ 2 , rue de la Butte verte \\ 93160 Noisy-le-Grand, FRANCE \\ Internet: mohri@univ-mlv.fr
}

\begin{abstract}
Finite-state transducers give efficient representations of many Natural Language phenomena. They allow to account for complex lexicon restrictions encountered, without involving the use of a large set of complex rules difficult to analyze. We here show that these representations can be made very compact, indicate how to perform the corresponding minimization, and point out interesting linguistic side-effects of this operation.
\end{abstract}

\section{MOTIVATION}

Finite-state transducers constitute appropriate representations of Natural Language phenomena. Indeed, they have been shown to be sufficient tools to describe morphological and phonetic forms of a language (Karttunen et al., 1992; Kay and Kaplan, 1994). Transducers can then be viewed as functions which map lexical representations to the surface forms, or inflected forms to their phonetic pronunciations, and vice versa. They allow to avoid the use of a great set of complex rules often difficult to check, handle, or even understand.

Finite-state automata and transducers can also be used to represent the syntactic constraints of languages such as English or French (Koskenniemi, 1990; Mohri, 1993; Pereira, 1991; Roche, 1993). The syntactic analysis can then be reduced to performing the intersection of two automata, or to the application of a transducer to an automaton. However, whereas first results show that the size of the syntactic transducer exceeds several hundreds of thousands of states, no upper bound has been proposed for it, as the representation of all syntactic entries has not been done yet. Thus, one may ask whether such representations could succeed on a large scale.

It is therefore crucial to control or to limit the size of these transducers in order to avoid a blow up. Classic minimization algorithms permit to reduce to the minimal the size of a deterministic automaton recognizing a given language (Aho et al., 1974). No similar algorithm has been proposed in the case of sequential transducers, namely transducers whose associated input automata are deterministic.

We here briefly describe an algorithm which allows to compute a minimal transducer, namely one with the least number of states, from a given subsequential transducer. In addition to the desired property of minimization, the transducer obtained in such a way has interesting linguistic properties that we shall indicate. We have fully implemented and experimented this algorithm in the case of large scale dictionaries. In the last section, we shall describe experiments and corresponding results. They show this algorithm to be very efficient.

\section{ALGORITHM}

Our algorithm can be applied to any sequential transducer $T=(V, i, F, A, B, \delta, \sigma)$ where: $V$ is the set of the states of $T, i$ its initial state, $F$ the set of its final states, $A$ and $B$ respectively the input and output alphabet of the transducer, $\delta$ the state transition function which maps $V \times A$ to $V$, and $\sigma$ the output function which maps $V \times A$ to $B^{*}$. With this definition, input labels are elements of the alphabet, whereas output labels can be words. Figure 1 gives an example of a sequential transducer.

Transducers can be considered as automata over the alphabet $A \times B^{*}$. Thus, considered as such they can be submitted to the minimization in the sense of automata. Notice however that the application of the minimization algorithm for automata does not permit to reduce the number of states of the transducer $T$. We shall describe in the following how the algorithm we propose allows to reduce the number of states of this transducer.

This algorithm works in two stages. The first one modifies only the output automaton associated with the given sequential transducer $T$. Thus, we can denote by $\left(V, i, F, A, B, \delta, \sigma_{2}\right)$ the trans- 


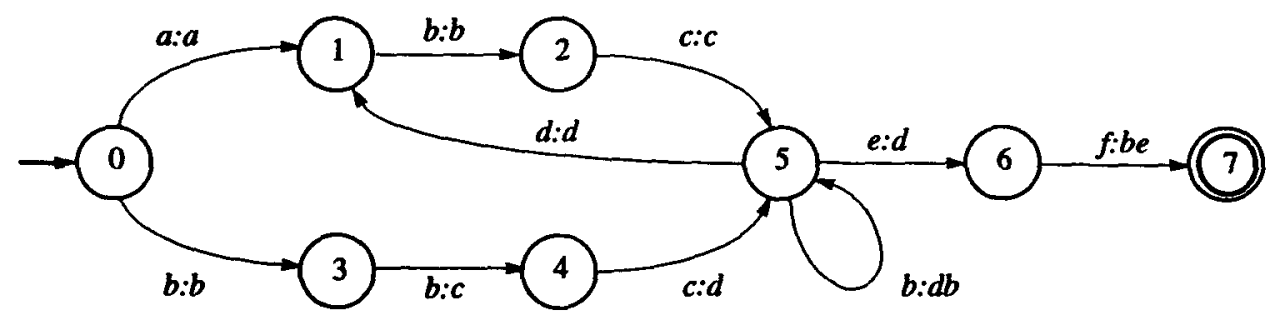

Figure 1. Transducer $T$.

ducer $T_{2}$ obtained after this first stage. Let $P$ be the function which maps $V$ to $B^{*}$ which associates with each state $q$ of $T$ the greatest common prefix of all the words which can be read on the output labels of $T$ from $q$ to a final state. The value of $P(5)$ is for instance $d b$ since this is the greatest common prefix of the labels of all output paths leaving 3. In particular, if $q$ is a final state then $P(q)$ is the empty word $\epsilon$. In order to simplify this presentation, we shall assume in the following that $P(i)=\epsilon$. The output function $\sigma_{2}$ of $T_{2}$ is defined by:

$$
\begin{array}{ll}
\forall q \in V, & \forall a \in A, \\
& \sigma_{2}(q, a)=(P(q))^{-1} \sigma(q, a) P(\delta(q, a)) .
\end{array}
$$

Namely, the output labels of $T$ are modified in such a way that they include every letter which would necessarily be read later on the following transitions. Figure 2 illustrates these modifications.
$T$ if beginning with the transition $(0,1)$. The output label of the following transition of $T_{2}$ is now empty. Indeed, anything which could be read from the transition $(1,2)$ on the output labels has now been included in the previous transition $(0,1)$.

It is easy to show that the transducer $T_{2}$ obtained after the first stage is equivalent to $T$. Namely, these two transducers correspond to the same function mapping $A^{*}$ to $B^{*}$. One may notice, however, that unlike $T$ this transducer can be minimized in the sense of automata and that this leads to a transducer with only six states. Figure 3 indicates the transducer $T_{3}$ obtained in such a way.

The second stage of our algorithm precisely consists of the application of the minimization in the sense of automata, that is, of merging equivalent states of the transducer. It can be showed that the application of the two presented stages to



Figure 2. Transducer $T_{2}$.

It shows the transducer $T_{2}$ obtained from $T$ by performing the operations described above. Notice that only the output labels of $T$ have' been modified. The output label $a$ corresponding to the transition linking states 0 and 1 of the transducer has now become $a b c d b$ as this is the longest word which is necessarily read from the initial state 0 of a sequential transducer $T$ systematically leads to an equivalent sequential transducer with the minimal number of states (Mohri, 1994). Indeed, the states of this minimal transducer can be characterized by the following equivalence relation: two states of a sequential transducer are equivalent if and only if one can read the same words from 


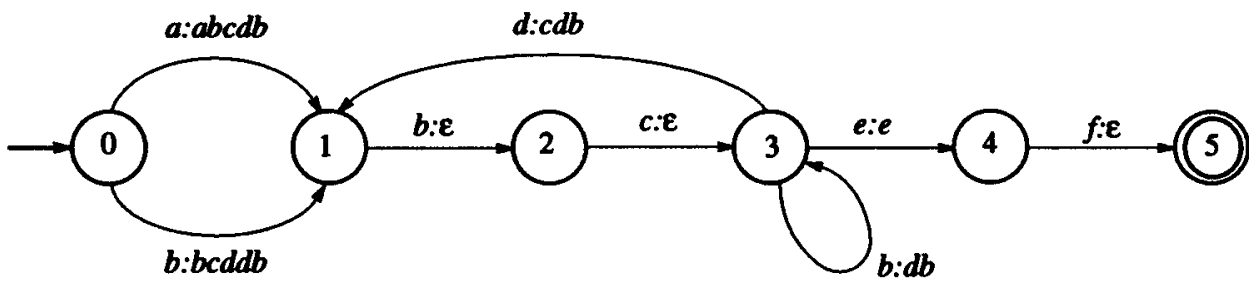

Figure 3. Transducer $\boldsymbol{T}_{\mathbf{3}}$.

these states using the left automaton associated with this transducer (equivalence in the sense of automata) and if the corresponding outputs from these states differ by the same prefix for any word leading to a final state. Thus, the described algorithm can be considered as optimal.

Notice that we here only considered sequential transducers, but not all transducers representing sequential functions are sequential. However, transducers which are not sequential though representing a sequential function can be determinized using a procedure close to the one used for the determinization of automata. The algorithm above can then be applied to such determinized transducers.

The complexity of the application of a non sequential transducer to a string is not linear. This is not the case even for non-deterministic automata. Indeed, recognizing a word $w$ with a non-deterministic automaton of $|V|$ states each containing at most $e$ leaving transitions requires $O(e|V||w|)$ (see Aho et al., 1974). The application of a non-sequential transducer is even more time consuming, so the determinization of transducers clearly improves their application. We have considered above sequential transducers, but transducers can be used in two ways. These transducers, although they allow linear time application on left, are generally not sequential considered as right input transducers. However, the first stage of the presented algorithm constitutes a pseudodeterminization of right input transducers. Indeed, as right labels (outputs) are brought closer to the initial state as much as possible, irrelevant paths are sooner rejected.

Consider for example the string $x=a b c d b c d b e$ and compare the application of transducers $T$ and $T_{2}$ to this sequence on right input. Using the transducer $T$, the first three letters of this sequence lead to the single state 5 , but then reading $d b$ leads to a set of states $\{1,5,6\}$. Thus, in order to proceed with the recognition, one needs to store this set and consider all possible transitions or paths from its states. Using the transducer $T_{2}$ and reading $a b c d b$ give the single state 1 . Hence, although the right input transducer is not sequential, it still permits to reduce the number of paths and states to visit. This can be considered as another advantage of the method proposed for the minimization of sequential transducers: not only the transducer is sequential and minimal on one side, but it is also pseudo-sequential on the other side.

The representation of language often reveals ambiguities. The sequential transducers we have just described do not allow them. However, real ambiguities encountered in Natural Language Processing can be assumed to be finite and bounded by an integer $p$. The use of the algorithm above can be easily extended to the case of subsequential transducers and even to a larger category of transducers which can represent ambiguities and which we shall call p-subsequential transducers. These transducers are provided with $p$ final functions $\phi_{i}$, $(i \in[1, p])$ mapping $F$, the set of final states, to $B^{*}$. Figure 4 gives an example of a 2-subsequential transducer.

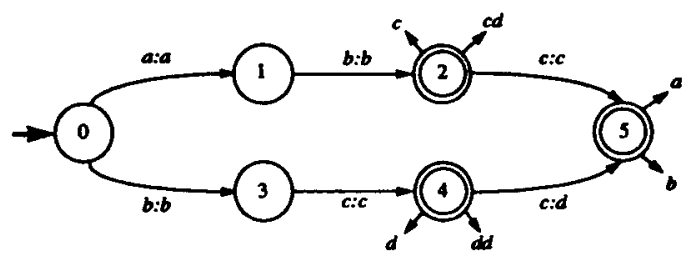

Figure 4. 2-subsequential transducer $T_{4}$.

The application of these transducers to a string $x$ is similar to the one generally used for sequential ones. It outputs a string corresponding to the concatenation of consecutive labels encoutered. However, the output string obtained once reaching state $q$ must here be completed by the $\phi_{i}(q)$ without reading any additional input letter. The application of the transducer $T_{4}$ to the word $a b c$ for instance provides the two outputs $a b c a$ and $a b c b$.

The extension of the use of the algorithm above is easy. Indeed, in all cases $p$-subsequential 
transducers can be transformed into sequential transducers by adding $p$ new letters to the alphabet $A$, and by replacing the $p$ final functions by transitions labeled with these new letters on input and the corresponding values of the functions on output. These transitions would leave the final states and reach a newly created state which would become the single final state of the transducer. The minimal transducer associated with the 2subsequential transducer $T_{4}$ is shown on figure 5 . It results from $T_{4}$ by merging the states 2 and 4 after the first stage of pseudo-determinization.

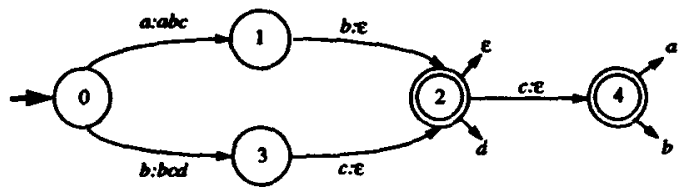

Figure 5. Minimal 2-subsequential transducer $T_{5}$.

In the following section, we shall describe some of the experiments we carried out and the corresponding results. These experiments use the notion of $p$-subsequential transducers just developped as they all deal with cases where ambiguities appear.

\section{EXPERIMENTS, RESULTS, AND PROPERTIES}

We have experimented the algorithm described above by applying it to several large scale dictionaries. We have applied it to the transducer which associates with each French word the set of its phonetic pronunciations. This transducer can be built from a dictionary (DELAPF) of inflected forms of French, each followed by its pronunciations (Laporte, 1988). It can be easily transformed into a sequential or $p$-subsequential transducer, where $p$, the maximum number of ambiguities for this transducer, is about four (about $\mathbf{3 0}$ words admit 4 different pronunciations). This requires that the transducer be kept deterministic while new associations are added to it.

The dictionary contains about 480.000 entries of words and phonetic pronunciations and its size is about $10 \mathrm{Mb}$. The whole minimization algorithm, including building the transducer from the dictionary and the compression of the final transducer, was quite fast: it took about 9 minutes using a HP 9000/755 with $128 \mathrm{Mb}$ of RAM. The resulting transducer contains about 47.000 states and 130.000 transitions. Since it is sequential, it can be better compressed as one only needs to store the set of its transitions. The minimal transducer obtained has been put in a compact form occupying about 1,1 Mb. Also, as the transducer is sequential, it allows faster recognition times.

In addition to the above results, the transducer obtained by this algorithm has interesting properties. Indeed, when applied to an input word $w$ which may not be a French word this transducer outputs the longest common prefix of the phonetic transcriptions of all words beginning with $w$. The input $w=$ opio for instance, though it does not constitute a French word, yields opjoman. Also, $w=$ opht gives oftalm. This property of minimal transducers as defined above could be used in applications such as OCR or spellchecking, in order to restore the correct form of a word from its beginning, or from the beginning of its pronunciation.

Table 1. Results of minimization experiments

\begin{tabular}{||l|c|c|c||}
\hline & DELAPF & FDELAF & EDELAF \\
\hline Initial size & $9,6 \mathrm{Mb}$ & $22,3 \mathrm{Mb}$ & $3,5 \mathrm{Mb}$ \\
\hline Entries & 480.000 & 780.000 & 145.000 \\
\hline Max. ambg & 4 & 15 & 8 \\
\hline Final size & $1,1 \mathrm{Mb}$ & $1,6 \mathrm{Mb}$ & $1 \mathrm{Mb}$ \\
\hline States & 47.000 & 66.000 & 47.000 \\
\hline Transitions & 130.000 & 195.000 & 115.000 \\
\hline Alphabet & 13.500 & 20.000 & 14.000 \\
\hline Time spent & $9^{\prime}$ & $20^{\prime}$ & $7^{\prime}$ \\
\hline
\end{tabular}

We have also performed the same experiment using 2 other large dictionaries: French (FDELAF) (Courtois, 1989) and English (EDELAF) (Klarsfeld, 1991) dictionaries of inflected forms. These dictionaries are made of associations of inflected forms and their corresponding canonical representations. It took about 20 minutes constructing the 15 -subsequential transducer associated with the French dictionary of about $22 \mathrm{Mb}$. Here again, properties of the obtained transducers seem interesting for various applications. Given the input $w=$ transducte for instance the transducer provides the output transducteur.N1:m. Thus, although $w$ is not a correct French word, it provides two additional letters completing this word, and indicates that it is a masculine noun. Notice that no information is given about the number of this noun as it can be completed by an ending $s$ or not. Analogous results were obtained using the English dictionary. A part of them is illustrated by the table above. It allows to compare the initial size of the file representing these dictionaries and the size of the equivalent transducers in memory (final size). The third line of the table gives the maximum number of lexical ambiguities encountered in each dictionary. The following lines indicate the number 
of states and transitions of the transducers and also the size of the alphabet needed to represent the output labels. These experiments show that this size remains small compared to the number of transitions. Hence, the use of an additional alphabet does not increase noticeably the size of the transducer. Also notice that the time indicated corresponds to the entire process of transformation of the file dictionaries into tranducers. This includes of course the time spent for I/O's. We have not tried to optimize these results. Several available methods should help both to reduce the size of the obtained transducers and the time spent for the algorithm.

\section{CONCLUSION}

We have informally described an algorithm which allows to compact sequential transducers used in the description of language. Experiments on large scale dictionaries have proved this algorithm to be efficient. In addition to its use in several applications, it could help to limit the growth of the size of the representations of syntactic constraints.

\section{REFERENCES}

Aho, Alfred, John Hopcroft, Jeffery Ullman. 1974. The design and analysis of computer algorithms. Reading, Mass.: Addison Wesley.

Courtois, Blandine. 1989. DELAS: Dictionnaire Electronique du $L A D L$ pour les mots simples du franais Technical Report, LADL, Paris, France.

Karttunen, Laura, Ronald M. Kaplan, and Annie Zaenen. 1992. Two-level Morphology with Composition. Proceedings of the fifteenth International Conference on Computational Linguistics (COLING'92), Nantes, France, August.

Kay, Martin, and Ronald M. Kaplan. 1994. Regular Models of Phonological Rule Systems. To appear in Computational Linguistics.

Klarsfeld, Gaby. 1991. Dictionnaire morphologique de l'anglais. Technical Report, LADL, Paris, France.

Koskenniemi Kimmo. $1990 . \quad$ Finite-state Parsing and Disambiguation. Proceedings of the thirteenth International Conference on Computational Linguistics (COLING'90), Helsinki, Finland.

Laporte, Eric. 1988. Méthodes algorithmiques et lexicales de phonétisation de textes. Ph.D the- sis, Université Paris 7, Paris, France.

Mohri, Mehryar. 1993. Analyse et représentation par automates de structures syntaxiques composées. Ph.D thesis, Université Paris 7, Paris, France.

Mohri, Mehryar. 1994. Minimization of Sequential Transducers. Proceedings of Combinatorial Pattern Matchnig (CPM'94), Springer-Verlag, Berlin Heidelberg New York. Also Submitted to Theoretical Computer Science.

Pereira, Fernando C. N. 1991. FiniteState Approximation of Phrase Structure Grammars. Proceedings of the 29th Annual Meeting of the Association for Computational Linguistics (ACL'91), Berkeley, California.

Roche Emmanuel. 1993. Analyse syntaxique transformationnelle du français par transducteur et lexique-grammaire. $\mathrm{Ph} . \mathrm{D}$ thesis, Université Paris 7, Paris, France. 
Mehryar MOHRI

Institut Gaspard Monge

Université Marne-la-Vallée

2 , Rue de la Butte verte

93166 NOISY-LE-GRAND CEDEX

FRANCE

Ph: $\begin{array}{llllll}33 & \text { (1) } & 49 & 32 & 60 & 54\end{array}$

Fax: 33 (1) $43 \quad 04 \quad 1605$ 